\title{
FEM STUDY TO ANALYZE THE EFFECT OF VIBRATIONS ON HUMAN BODY JOINTS
}

\author{
Akash Bhatia $^{1}$, Anuj Sehgal ${ }^{2}$, Sachin Kalsi ${ }^{3}$ \\ ${ }^{1}$ Research Scholar, Department of Mechanical Engineering, Chandigarh University, Punjab \\ ${ }^{2}$ Professor, Department of Mechanical Engineering, Chandigarh University, Punjab \\ ${ }^{3}$ Assistant Professor, Department of Mechanical Engineering, Chandigarh University, Punjab \\ Corresponding Author: phd.sachinkalsi@gmail.com
}

DOI: $10.51201 / J U S S T / 21 / 05202$

$$
\text { http://doi.org/10.51201/JUSST/21/05202 }
$$

Abstract: Whenever a human body stands on a platform like bus, train etc then the waveform, frequency, amplitude and duration due to vibrations can be changing and these variations produce some advantages in muscles and bone mineral density whereas high acceleration of platform viewed as dangerous. The reason behind this is the resonance of human body that depends upon some factors: the seat surface material, the posture, vibration magnitude and frequency etc. In this study, modal and harmonic response analysis has been performed to calculate the natural frequencies of Indian human male subject in standing posture condition under un-damped and damped free vibration conditions. With the help of this we are capable to know how these vibrations effect our muscles, bones, joints etc. Joint studies of human body helps us to replace body joints in case of any serious injury or in emergency and multibody dynamics, modal analysis and harmonic response plays crucial role in these effects. This study shows the vibration effects of transmissibility and stresses on knee joints and neck joint of human body. For this purpose a revolute joint is separately designed and fixed at the location of joints in human subject to understand the effect of these vibrations. The results are determined in this study is within the frequency range of 0-20 $\mathrm{Hz}$.

\section{Keywords: FEM, Modal Analysis, Harmonic Analysis, Human Joints.}

\section{INTRODUCTION}

With the advancement in technology, develop high-tech machines for the reason that can improve and make our lifestyle comfortable. As it has been seen whenever a person drive a car, taxi, bus etc there feel sometime uncomfortable, back pain at lower spine, visual fatigue and motion sickness etc and the major cause of these problems are vibrations. There are so many proved studies which show that how vibrations effects human body whereas some studies also proved that whole body vibration is the effective rehabilitation tool. Whenever a human body stands on a platform which vibrates like in bus, train etc then the waveform, frequency, amplitude and duration can be changes and these variations produce some advantages in muscles and bone mineral density whereas high acceleration of platform viewed as dangerous. The reason behind this is the resonance generates in human body which depends upon some factors: the material of the seat surface, the posture, frequency and vibration magnitude etc. Simulation studies with the help whole body vibrations, multibody dynamics and finite element method also prove these effects and make us easy to understand visually with the help of computer software. The measures are stated in terms of force-motion relation, apparent mass, absorbed power, mechanical impedance and flow of vibration through human subject i.e seat to head (STHT) vibration transmissibility. There are number of researchers who perform research in this area, Crewther et al. [1] determines the gravitational forces at different body postures and also at different amplitudes, frequencies and anatomical sites. This study, collapses whole data into four different categories (posture, frequency, damping and amplitude) and find the gravitational forces in each category. After analysis research shows that gravitational forces associated with semi-squat posture $(2.34 \mathrm{~g})$ were greater $(\mathrm{P}<0.001)$ as compared to standing posture of human body. The gravitational forces decreased with increasing gap between the vibrating surface $(\mathrm{F}=283.7, \mathrm{P}<0: 001)$. The power of this test with $\alpha=0.050$ was 1.00 . Bei et al. [2] present the study which proves that multibody dynamics musculoskeletal models are appropriate for predicting muscle forces and joint pressures at the same time and it is helpful to study the clinical issues related to restoration and degeneration of knees. This work represents the combined computational method for deformable contact knee model and multibody dynamics. The natural knee model for static analysis is used in this research is created with the help MRI, CT scan and 
manufacture's CAD data. It predicts same amount of torque and forces but different contact pressures for linear models and for non linear models the effect is lower because of increased contact area in case of dynamic simulations of knee models. Veloso et al. [3] conducted a software simulation of mechanical model created with the help of MSC Adams Life modeler for a workstation where driving of electric screw is used. This study helps to understand the estimation of the loads of muscle skeletal in human activities in the car industry. The data used to create this model is provided by electromyography measurements, video recordings and anthropometric data of workers. The results evaluated from this research is validated with the help of electromyography comparisons. Teng et al. [4] proposed a research on how vibrations affects human body under various conditions which is the cause of low back pain, spinal injury, uncomfortable feelings etc. The understanding of these affects may helps to reduce these injuries. This study represents a seated human body subject under the vibrating conditions evaluated with the help of multibody dynamics. The equations of motion in this dynamic system of mechanical structure are based on Kane's equation and then it is coded into the computer programme and solved with the help of Range Kutta Method of fourth order. This analysis is based on two siting postures erect and relaxed and also finds that $5 \mathrm{~Hz}$ frequency should be avoided for the sitting posture having no support. This article also explains the peak values at resonance frequencies are lower in case of erect posture as compared to relaxed posture resonance frequencies. Nazer et al. [5] proposed a research which estimates the tibial strains which occurs during walking of human body with the help of numerical approach using flexible multibody dynamics. For this purpose a lower body musculoskeletal model was created by implementing the data from motion capture device which is obtained during walking condition of human body at constant velocity. The data from motion capture device helpful in inverse dynamic simulations for the purpose of replication of motion in forward dynamics simulation. We get the minimum and maximum tibial strains 490 and -588 micro strains in the form of results from this model. Subashi et al. [6] proposed a study which proves the effect of resonances on the human body standing in different five postures i.e upright, knees bent, knees more bent, lordotic and anterior lean. For this purpose a lumped parameter mathematical model which represents the anatomical parts of a human body was created to show the human body motion which associates with the resonances of vertical apparent and for-and-aft cross-action apparent mass of a human body. All the parameters either it is geometric and inertial were obtained for created model from the published anthropometric data. The results provided by this study is based on the behaviour and effects of the vibration and resonances under the different five standing conditions. Milosavljevic et al. [7] proposed a study which shows that how whole body vibrations effects the farmers when they use all-terrian vehicle on daily basis. For the purpose of study these effects this research follows guidelines given by the International Organization for Standardization (ISO). In order to obtain the daily vibration dose value the frequency weighted vibration exposure and total time of riding was calculated. Some values of vibration and shocks was set by the ISO for the purpose of reduction of this effect. This study also finds the results when all the participants are exposed to the exceeded limit of vibration and shocks as compared to the ISO recommendations under different seasonal conditions and it finds that low back pain problem is mostly reported. Rakheja et al. [8] present a study which shows that the identification of the ranges of biodynamic response of human body during the exposure of whole body vibrations and it is very much needed because it helps to develop effective and ergonomically improved machines for humans, improved devices which are capable to absorb vibrations and design tools. For this purpose in this work ranges for the biodynamic responses were derived for the body seated with backrest, without backrest and also for standing conditions. The identification of these ranges of biodynamic responses is targeted to the design of essential anthrop dynamic manikins, generation of human body CAD models which helps to determine the induced stresses in the body joints and also for the purpose of calculating the alternate frequency weightings for judgment of vibration influence. Nawayseh and Griffin [9] proposed a study which investigated the magnitude of vibration on the power absorbed during whole body vibration when vibration affects the support system for feet and back. In this study twelve human subjects were influenced to four magnitudes $\left(0.125,0.250 .625\right.$ and $1.25 \mathrm{~m} / \mathrm{s}^{2} \mathrm{rms}$ ) of the vertical (in z-axis) vibrations $(0.25-20 \mathrm{~Hz})$ when sitting on the hard seat in four different postures (maximum thigh contact, average thigh contact, minimum thigh contact and feet hanging,) with hard backrest and without hard backrest. The acceleration and force was calculated at the feet, seat and backrest to determine the power absorbed at these points. The results showed that the power absorbed at all these three points (seat, backrest and feet) increased in the proportion of the square of the magnitude 
of vibration and the maximum power absorbed is at seat. Bressel et al. [10] proposed a study on the whole body vibration transmission in children when they are in standing position. The main purpose of this study is to analyze the transmission of vibration in children is different from adults when it stands on the platform which vibrates. Some recent studies proved that the children with disabling conditions are treated with the help of whole body vibrations. It is observed that children have the capability to attenuating the high vibrations due to less mass. In this study 11 children and 10 adults are needed to stand on the vibrating platform having greater frequencies(28, 33 and 42). The results which are determined from this study shows that transmissibility of whole body vibration in the case of standing is not different for adult and children. There is only one difference in the case of $33 \mathrm{~Hz}$ frequency the transmissibility of ankle and hip is higher in case of children as compared to adults which proved that this therapy is not safe for the children. Shabana et al. [11] present a study which shows the use of absolute nodal coordinate formulation in the integration of multibody system and finite element algorithm in biomechanics for modeling of rigid body contacts and ligaments flexibility. It develops a nonlinear algebraic equations which are based on surface parameters. These equations are calculated iteratively for the purpose to calculate the location of contact points. In this investigation a computational algorithm created and represented using a knee joint model in which a linear spring damper element is used for modeled ACL and PCL whereas the large displacement ANCF finite element is used for modeled LCL and MCL which helps to allow for the use of general constitutive models and capture deformation in the ligament cross-section. Liu et al. [12] proposed a study in which a multibody numerical model having three dimensions is generated with the help of Life MOD and ADAMS is presented to analyze and simulate the load under bicycling conditions of model in which wrist, leg muscles, ankles, shoulder and knees are considered. In three dimensional multibody model horizontal and vertical position of saddle are adjusted according requirements to simulate muscle forces for race and city bicycle riding. The main objective of this study to determine a better posture of bicycle riding either for race or city to prevent sports person from sports injuries. The results of this study shows the different effects of vibrations at different body postures while riding. Guo et al. [13] present a study which is designed for the investigation of the model behaviour of human spine. In this study a three dimensional human spine T12-pelvis segment model was used for the extraction of resonant frequencies. In the analysis of harmonic response and finite element model so many lower vibration modes in the vertical directions, flexion-extension and lateral bending were determined and its vibrational configuration was shown in this research. This configuration of lumber spine can investigate the motion mechanism of lumber components under the effect of whole body vibrations and helps us to understand the diseases which is induced due to the vibrations in the human spine. Ambrosio et al. [14] proposed a study which investigate the behaviour of upper limb of the musculoskeletal system. This consists total of three musculoskeletal model of multibody formulations were considered. The main purpose of this work is to compare the availability and performance of all the three biomechanical models under different stages of complexity. The whole data was scaled to our skeletal system making a total of 20 muscles modeled by 127 bundles. The simulation of dynamics of muscle contraction was done with the help of Hill type muscle model. In this research the indeterminacy is removed with the help of optimization technique is implemented through the minimization of objective function which is directly related with the energy consumption of muscle metabolics. Klodowski et al. [15] conducted a study on lower body skeletal loading analysis with the use of flexible multibody dynamics approach. This approach helps to allow for investigating the skeletal loading and strains between the bones in which ground reaction forces, muscle force, natural motion of a subject and bone flexibility is accounted. This research represents how standard multibody formulations can be introduced by deformable bodies and also helps to determine the benefits and drawbacks. The comparison of results for this simulation is with in-vivo studies and shows acceptable method performance and correlations. Duan et al. [16] present a study in which the mechanism of human body response understands under the exposure of vibration. A whole body vibration platform is needed which helps to give a source of vibrational interventions. A person is also needed who stands on this platform under the exposure of whole body vibrations. On the basis of this data a biomechanical human model is developed which represents the human body and whole body vibration platform. Kane's method is used to calculate equations of motions of this system. Basri et al. [17] conducted a research for predicting the discomfort when a person sitting under the inclined seat conditions $\left(0^{\circ}, 30^{\circ}, 60^{\circ}, 90^{\circ}\right)$ or no backrest conditions and exposed to whole body vibrations. Among of these different five postures, 12 subjects feels the discomfort due to whole body vibrations. In this study the points in the body 
where discomfort was experienced for each frequency they were determined at two vibration magnitudes. The study concludes that if the frequency is greater than $8 \mathrm{~Hz}$ the backrest increased vibration discomfort and specially for head and neck the discomfort value is high. At the frequency 5 and $6.3 \mathrm{~Hz}$ the value of discomfort was very less under the condition of inclined backrest. Uttam Y. and Samir B. [18] present a study in which natural frequency analysis of an automotive seating system with the help of finite element method for vibration concern. The aim of this study is only to check whether the specifications and requirements of OEM(original equipment manufacturer) of modal frequency management is meets or not. In this research a finite element model of a seat is prepared and analyze it for the natural frequencies according to the point of view of modal management and also have to find out critical frequencies and prevent it from the other components and human body so that resonance not occurred. Literature review shows that most of the work has been performed experimentally on cushion material, seat suspension and ergonomics of a vehicle as compared to Finite Element Method(FEM). There are very less availability of data which shows the effects of vibrations on human joints.

\section{METHODOLOGY}

Whenever a body stands on any vibrating platform it is affected by these vibrations causing joint pain, muscle pain, motion sickness etc. The main purpose of this work to study the biodynamic response of human joints and study its behavior when exposed to whole body vibrations. For this purpose a revolute joint is assigned to the human subject and study the behavior of knee and neck joint under the vibrating conditions when acceleration is $1 \mathrm{~m} / \mathrm{s}^{2}$.

\subsection{Generation of 3D CAD Model}

Computer Aided Design software is used in this work to make human subject model. All the body parts of human subject is designed separately in solidworks software and every dimension is given to this model is on the basis on anthropometric data. After the completion of the design of separate body parts it combines with the help of assembly module present in the solidworks. The 3D CAD model developed in solidworks software that has been shown in Figure 1 and then export as parasolid file, and while performing modal analysis, mesh has been generated using tetrahedral elements because tetrahedral elements are stiffer and gives better results in complex structures compared to other elements. During analysis, boundary conditions were taken by considering feet to be fixed with the floor and physical properties of human subject has been considered to be homogeneous and isotropic in nature. To complete this model a revolute joint is necessary which is also designed separately and assigned at every human body joint. After applying all the operations finally we get the assembled human subject and after that it exports it into Ansys 15.0 to study the modal and harmonic response.

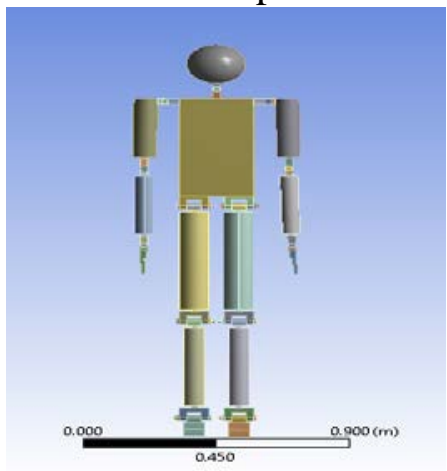

Figure 1. 3D CAD Model 


\subsection{Meshing of 3D CAD Model}

Meshing of CAD Model is done with the help of Ansys software and tetrahedron meshing is performed that has been shown in Figure 2. There are total of 35636 nodes and 16472 elements are present in the model after meshing. As it is much stiffer than other elements and is a better option for performing meshing off complex geometries. An element size in performing mesh for this human subject has been considered to be of $3 \mathrm{~mm}$.

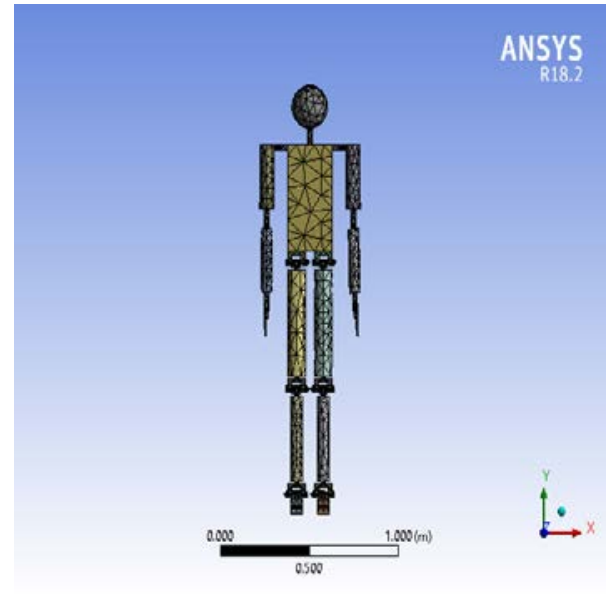

Figure 2. Meshing of CAD Model

\subsection{Boundary Conditions}

Human subject analysis in standing posture that is fixed from the feet as shown in the Figure 3. In the investigation of the vibration effects 0.3 damping ratio coefficient is taken and no external load is applied on this model. Only the vertical direction has been taken into consideration for excitation. Only $0-20 \mathrm{~Hz}$ frequency is taken under considerations for this work.

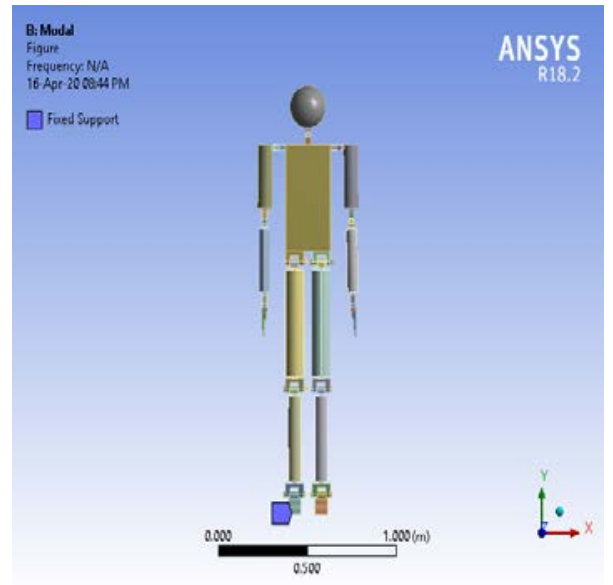

Figure 3. Fixed Support at the Feet of Human Subject

\section{RESULTS AND DISCUSSION}

\subsection{Modal Analysis}

The human body subject is then exposed to vibrations at different frequencies and performed a modal analysis with the help of Ansys Software 15.0 and study the total deformation occurs at different frequencies that has been shown in Table 1. 
Table 1. Human Subject Exposed to Vibrations at Different Frequencies.

\begin{tabular}{|c|c|c|}
\hline Sr. No. & Mode & Frequency $(\mathrm{Hz})$ \\
\hline 1. & 1. & 3.1536 \\
\hline 2. & 2. & 7.9773 \\
\hline 3. & 3. & 21.301 \\
\hline 4. & 4. & 26.031 \\
\hline 5. & 5. & 27.713 \\
\hline 6. & 6. & 28.893 \\
\hline
\end{tabular}

Frequencies shows in the Table 1are the 6 steps of frequencies which is applied to human subject one by one and find the respective results in the form of total deformation and stresses. All the outcomes added in Figure 4, 5, 6,7,8 and 9.

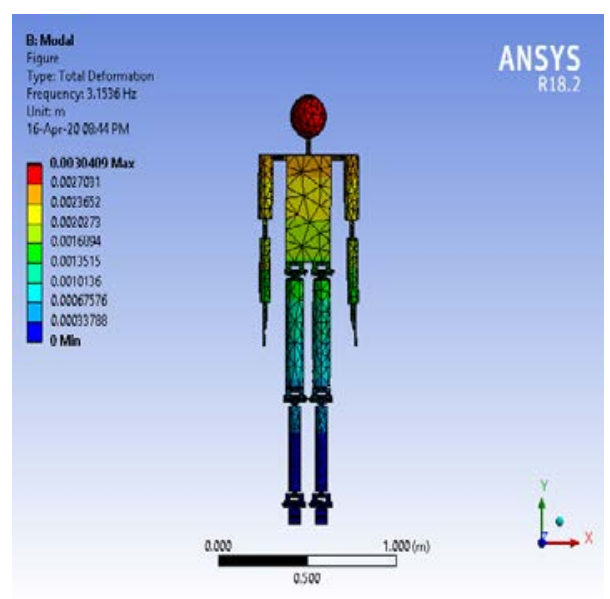

Figure 4. Total Deformation at Frequency of $3.1536 \mathrm{~Hz}$.

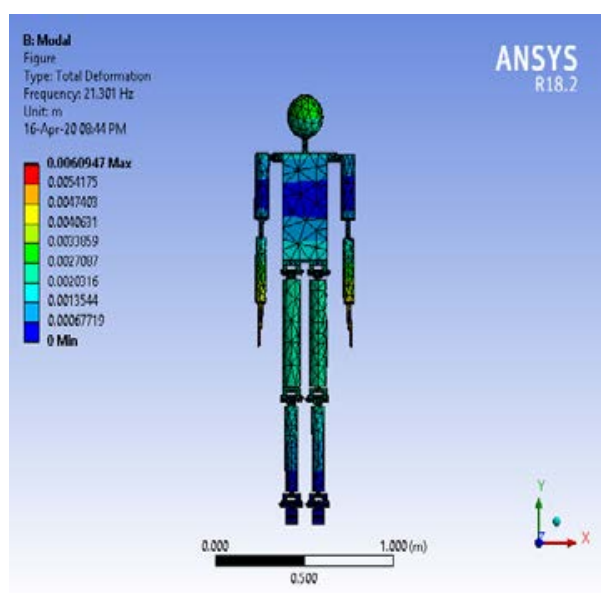

Figure 6. Total Deformation at Frequency of $21.301 \mathrm{~Hz}$.

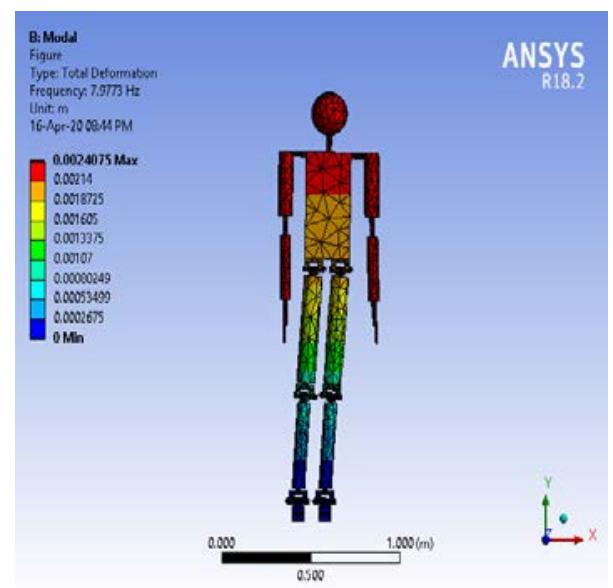

Figure 5. Total Deformation at Frequency of $7.9773 \mathrm{~Hz}$.

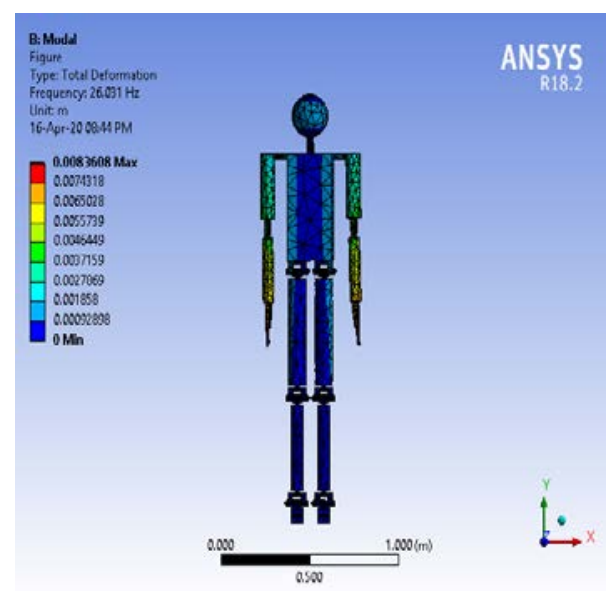

Figure 7. Total Deformation at Frequency of $26.031 \mathrm{~Hz}$. 


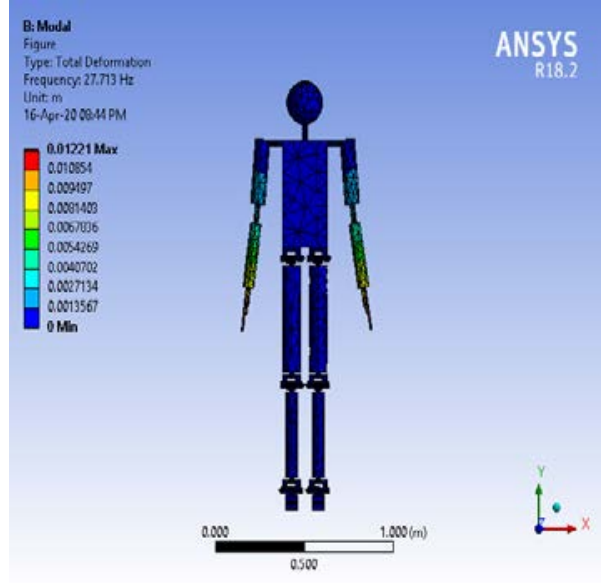

Figure 8. Total Deformation at Frequency of $27.713 \mathrm{~Hz}$.

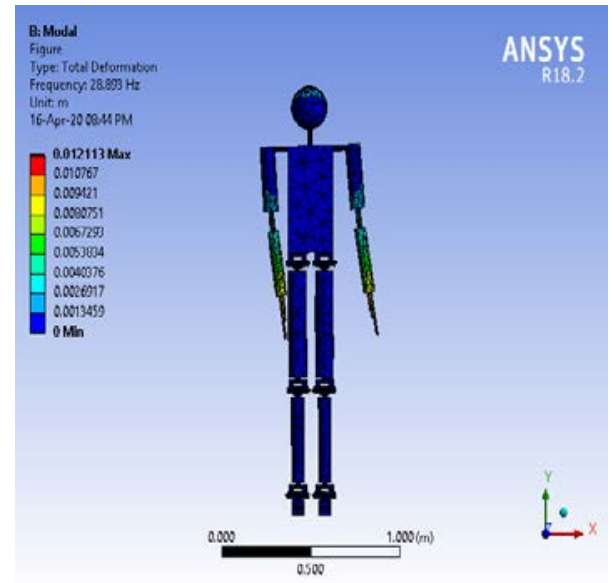

Figure 9. Total Deformation at Frequency of $28.893 \mathrm{~Hz}$.

\subsection{Harmonic Analysis}

Harmonic analysis of human subject is performed to understand the effect of vibrations at Head, knee joints and neck joint because when a human body is in standing posture then whole body weight in focused at the knee joints and at the same time it exposed to the vibrations then it is the cause of serious injury and that effects we studied in this research with the help of Modal analysis and Harmonic response. It also helps to evaluate the transmissibility and stresses induced in the respective joints as shown below.

Table 2. Represents the Transmissibility Values at Head.

\begin{tabular}{|c|c|c|}
\hline Sr. No. & Frequency (Hz) & Transmissibility $\mathbf{( m / \mathbf { s } ^ { 2 } )}$ \\
\hline 1. & 2 & 1.0764 \\
\hline 2. & 4 & 1.3384 \\
\hline 3. & 6 & 1.8768 \\
\hline 4. & 8 & 2.162 \\
\hline 5. & 10 & 1.5362 \\
\hline 6. & 12 & 1.0449 \\
\hline 7. & 14 & 0.77676 \\
\hline 8. & 16 & 0.61965 \\
\hline 9. & 18 & 0.51881 \\
\hline 10. & 20 & 0.44942 \\
\hline
\end{tabular}

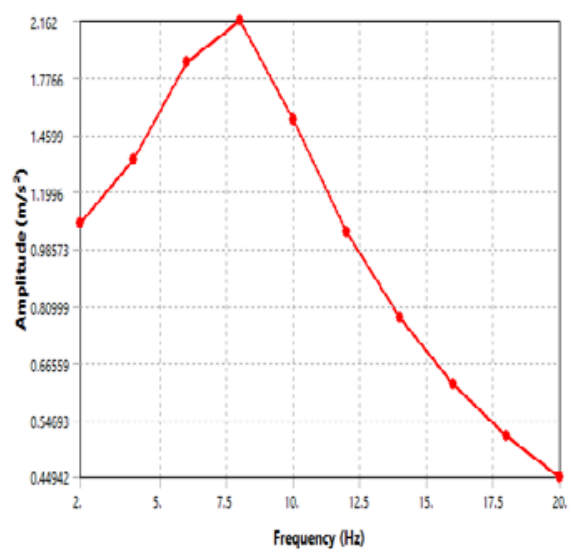

Figure 10. Graph of Frequency Response at Head.

Table 2 represents the transmissibility values at head and figure 10 represents the graph of frequency response at head. These outcomes shows that the maximum value of transmissibility is $2.162 \mathrm{~m} / \mathrm{s}^{2}$ at $8 \mathrm{~Hz}$ frequency and also represents that the transmissibility value decreases as the frequency increases after the peak value. 
Table 3. Represents the Stresses Induced at the Knee Joints

\begin{tabular}{|c|c|c|}
\hline Sr. No. & Frequency (Hz) & Stress (Pa) \\
\hline 1. & 2 & 639.77 \\
\hline 2. & 4 & 498.86 \\
\hline 3. & 6 & 597.44 \\
\hline 4. & 8 & 551.19 \\
\hline 5. & 10 & 301.29 \\
\hline 6. & 12 & 185.86 \\
\hline 7. & 14 & 175.58 \\
\hline 8. & 16 & 198.98 \\
\hline 9. & 18 & 225.69 \\
\hline 10. & 20 & 243.64 \\
\hline
\end{tabular}

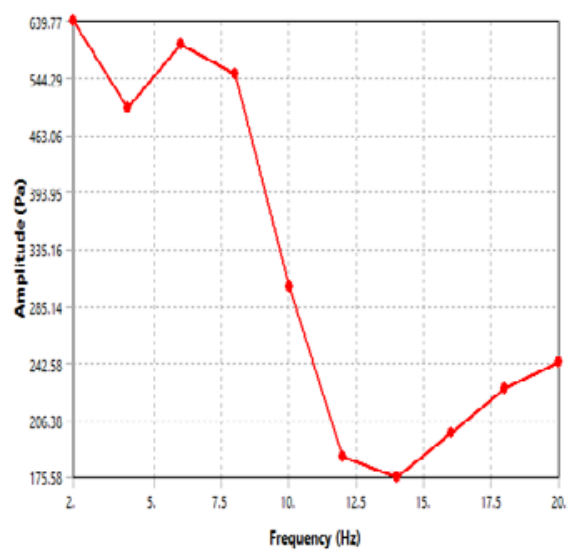

Figure 11. Graph of Stresses Induced at Knee Joints

Table 3 shows the stresses induced at knee joints and Figure 11 is the graphical representation of stresses induced at knee joints. According to the results the maximum stress value is 639.77 pa induced at $2 \mathrm{~Hz}$ and minimum stress value is 175.58 pa and induced at $14 \mathrm{~Hz}$.

Table 4. Represents the Stresses Induced at Neck Joints.

\begin{tabular}{|c|c|c|}
\hline Sr. No. & Frequency (Hz) & Stress (Pa) \\
\hline 1. & 2 & 33763 \\
\hline 2. & 4 & 51230 \\
\hline 3. & 6 & 40133 \\
\hline 4. & 8 & 35703 \\
\hline 5. & 10 & 22296 \\
\hline 6. & 12 & 14233 \\
\hline 7. & 14 & 10332 \\
\hline 8. & 16 & 8205.1 \\
\hline 9. & 18 & 6765.7 \\
\hline 10. & 20 & 5473.5 \\
\hline
\end{tabular}

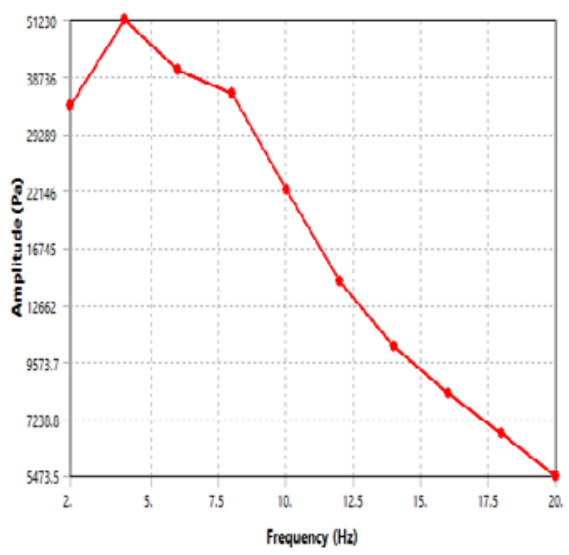


Figure 12. Graph of Stresses Induced at Neck Joint

Table 4 shows the stresses induced at neck joint and Figure 12 is the graphical representation of stresses induced at neck joint. It shows that the maximum stress value induced at $4 \mathrm{~Hz}$ i.e 51,230 pascal and minimum stress value induced at $20 \mathrm{~Hz}$ i.e 5,4730.5 pascal. The results shows that the value of stresses induced are decreases as the frequency increases.

\section{CONCLUSION}

Analysis of human subject in standing conditions are evaluated in this study and we find the effect of vibrations at joints because whenever a person is in standing posture either in bus and train it exposed to vibrations which causes a serious injury because at the same time whole body weight is focused at joints and this study also shows that how much our neck joint and knee joints are effected in this condition and also evaluate how much stresses are induced at different frequencies in the joints (knee joints and neck joint) of human subject. The value of transmissibility is also determined at different frequencies at head in this work. Different cases of damping have been considered while performing harmonic response analysis of human subject in the range of 0 to $20 \mathrm{~Hz}$ with acceleration of $1 \mathrm{~m} / \mathrm{s}^{2}$. Results obtained in current study for modal analysis of 3D CAD model of human body has been validated with the existing literature. From results it has been observed that, at low frequency maximum deformation is obtained at head. This work can be extended to find out the biodynamic response at joints of human body when exposed to vibrations during travelling by vehicle in sitting posture and also can be used to calculate the effect of vibrations at human body joints during the farm operations. The value of acceleration $1 \mathrm{~m} / \mathrm{s}^{2}$ and applied to the human subject in standing posture in between the frequency range of 0-20 $\mathrm{Hz}$, it can also be changed as per need for future work.

\section{REFERENCES}

1. Crewther, B., Cronin, J., \& Keogh, J. (2004). Gravitational forces and whole body vibration: implications for prescription of vibratory stimulation. Physical Therapy in Sport, 5(1), 37-43.

2. Bei, Y., \& Fregly, B. J. (2004). Multibody dynamic simulation of knee contact mechanics. Medical engineering \& physics, 26(9), 777-789.

3. Veloso, A., Esteves, G., Silva, S., Ferreira, C., \& Brandão, F. (2006, February). Biomechanics modeling of human musculoskeletal system using Adams multibody dynamics package. In Proceedings of the 24th IASTED International Conference on Biomedical Engineering, Innsbruck (pp. 401-407).

4. Teng, T. L., Chang, F. A., \& Peng, C. P. (2006). Analysis of human body response to vibration using multi-body dynamics method. Proceedings of the Institution of Mechanical Engineers, Part K: Journal of Multi-body Dynamics, 220(3), 191-202.

5. Al Nazer, R., Rantalainen, T., Heinonen, A., Sievänen, H., \& Mikkola, A. (2008). Flexible multibody simulation approach in the analysis of tibial strain during walking. Journal of biomechanics, 41(5), 1036-1043.

6. Subashi, G. H. M. J., Matsumoto, Y., \& Griffin, M. J. (2008). Modelling resonances of the standing body exposed to vertical whole-body vibration: Effects of posture. Journal of Sound and Vibration, 317(1-2), 400-418.

7. Milosavljevic, S., Bergman, F., Rehn, B., \& Carman, A. B. (2010). All-terrain vehicle use in agriculture: Exposure to whole body vibration and mechanical shock. Applied ergonomics, 41(4), 530-535.

8. Rakheja, S., Dong, R. G., Patra, S., Boileau, P. É., Marcotte, P., \& Warren, C. (2010). Biodynamics of the human body under whole-body vibration: Synthesis of the reported data. International Journal of Industrial Ergonomics, 40(6), 710-732. 
9. Nawayseh, N., \& Griffin, M. J. (2010). Power absorbed during whole-body vertical vibration: Effects of sitting posture, backrest, and footrest. Journal of Sound and Vibration, 329(14), 2928-2938.

10. Bressel, E., Smith, G., \& Branscomb, J. (2010). Transmission of whole body vibration in children while standing. Clinical biomechanics, 25(2), 181-186.

11. Shabana, A. A., Gantoi, F. M., \& Brown, M. A. (2011). Integration of finite element and multibody system algorithms for the analysis of human body motion. Procedia IUTAM, 2, 233-240.

12. Liu, Y. S., Tsay, T. S., \& Wang, T. C. (2011). Muscles force and joints load simulation of bicycle riding using multibody models. Procedia Engineering, 13, 81-87.

13. Guo, L. X., Zhang, Y. M., \& Zhang, M. (2011). Finite element modeling and modal analysis of the human spine vibration configuration. IEEE transactions on biomedical engineering, 58(10), 2987-2990.

14. Ambrósio, J., Quental, C., Pilarczyk, B., Folgado, J., \& Monteiro, J. (2011). Multibody biomechanical models of the upper limb. Procedia IUTAM, 2, 4-17.

15. Kłodowski, A., Rantalainen, T., Heinonen, A., Sievänen, H., \& Mikkola, A. (2011). The use of the flexible multibody approach for lower body skeletal loading analysis. Procedia IUTAM, 2, 93-100.

16. Duan, S., Mattison, L., \& Binkley, T. (2012, November). Multibody Dynamics Model for Analysis of Human Body Response to Vibrations. In ASME International Mechanical Engineering Congress and Exposition (Vol. 45202, pp. 1083-1088). American Society of Mechanical Engineers.

17. Basri, B., \& Griffin, M. J. (2013). Predicting discomfort from whole-body vertical vibration when sitting with an inclined backrest. Applied Ergonomics, 44(3), 423-434.

18. Siddha, U. Y., \& Kumbhar, S. B. (2013). Natural frequency analysis of automotive seating system by using FEM software. Int J Mech Engng Robotics, 1(2), 93-98. 